Mathematics

\section{Hermann Grassmann was right}

\section{from Ian Stewart}

THE late nineteenth century witnessed many attempts to develop an algebra of $n$-dimensional space, by analogy with the representation of the plane by complex numbers. Prominent among them was Hermann Grassmann's Die lineale Aus dehnungslehre (The Calculus of Extension), published in 1844 . Grassmann had the misfortune to write in a discursive, philosophical and obscure style at the time when axiomatic presentation was becoming de rigeur in the mathematical world. Further, he was a high-school teacher with no formal training in mathematics. Not surprisingly, his ideas were at first not taken very seriously - but later, Élie Cartan used Grassmann's 'exterior algebra' as the basis of his theory of differential forms. This work proved highly influential and established the importance of Grassmann's work. But, as Marielena Barnabei, Andrea Brini and Gian-Carlo Rota write in a recent paper (J. Algebra 96, 120; $1985)$ "In the process Cartan paid a price of omission from which we are yet to recover." They argue that mathematicians have still not fully understood what Grassmann was trying to say, and propose a formulation of his ideas in modern terms which provides a natural language for the study of invariants.

In Grassmann's original work the same notation was used to denote two different operations performed on vectors, called the progressive and regressive products. This was not an oversight, but a deliberate choice. Every mathematician knows that what Bourbaki calls "abuse of notation" can, when wielded with care, greatly illuminate what would otherwise be a confusing situation. Unfortunately Grassmann managed to confuse greatly what might otherwise have been an illuminating situation. The tale has two main threads: differential forms and invariants.

Cartan's theory of differential forms attempts to make sense of the idea that expressions such as $\mathrm{d} x$ and $\mathrm{d} y$ in calculus can have a perfectly sensible meaning. The intuitive idea of 'a very tiny bit of $x$ ' runs into severe difficulties, and is not very helpful; but the basic idea that the derivative $\mathrm{d} y / \mathrm{d} x$ satisfies an equation $\mathrm{d} y=(\mathrm{d} y / \mathrm{d} x) \mathrm{d} x$ is crucial. So is the idea that an integral $\int \mathrm{d} x$ can be thought of as an operation / applied to a differential form $\mathrm{d} x$. The fundamental observation is that products of differential forms, such as $\mathrm{d} x . \mathrm{d} y$, do not obey the usual laws of algebra. This is because the double integral $\int \mid \mathrm{d} x . \mathrm{d} y$ represents a signed area, positive or negative; and interchanging $x$ and $y$ turns a left-handed coordinate system into a right-handed one, multiplying all areas by -1 . In other words, one must set up the formation to make $\mathrm{d} x \cdot \mathrm{d} y=-\mathrm{d} y \cdot \mathrm{d} x$. Grassmann's progressive product obeys the same kind of law. Cartan found that the regressive product added nothing to his theory, and he quietly abandoned it. Until now, its demise has gone unmourned.

The new work of Barnabei et al. is part of an extensive programme of research that Rota has carried out for more than a decade, applying combinatorial methods to invariant theory. Classical invariant theory is the study of expressions that remain unchanged when their variables are transformed in particular ways. A simple example arises in connection with quadratic equations $a x^{2}+b x+c=0$, for which there is a well-known formula involving square roots of $b^{2}-4 a c$. This quantity is called the discriminant, because it vanishes precisely when the two roots of the equation are equal. Suppose the variable is changed from $x$ to $x+h$. Then the quadratic becomes $(x+h)^{2}+b(x$ $+h)+c$, or $a x^{2}+(2 a h+b) x+\left(a h^{2}+b h\right.$ $+c)$. Write this as $A x^{2}+B x+C$. Now compute the new discriminant: $B^{2}-4 A C=$ $(2 a h+b)^{2}-4 a\left(a h^{2}+b h+c\right)=b^{2}-4 a c$. Thus the formula for the discriminant does not change, although the variable $x$ does. It is called an invariant of the transformation.

Invariant theory was invented around 1850 by Arthur Cayley and James Joseph Sylvester. It considers more complicated expressions than only quadratics, and more complicated transformations than $x \rightarrow x+h$. The fundamental problem in the theory is to prove that, in any particular case, the full system of invariants can be expressed in the terms of a finite subsystem, called a basis. The main classical method is to describe explicitly all invariants and to exhibit such a basis. By such methods Paul Gordan, the 'king of the invariants', proved in 1868 that for any expression in two variables, a finite basis exists. Limited extensions were proved by Gordan and others, but progress became painfully slow as ideas were overwhelmed by a swelling mountain of calculation.

In 1888 David Hilbert created a tremendous stir by proving that a finite basis always exists - and he did it without calculating any invariants at all. He used only very general abstract properties of systems of polynomials. "This is not mathematics", Gordan complained, "it is theology". But theology won the day, and explicit computation of invariants went out of fashion, being replaced by a much more abstract, higher level approach that gave conceptual ideas pride of place. But history tends to run in spirals; and in recent decades there has been something of a classical revival, inspired in part by the needs of other branches of mathematics, and by applications. Explicit formulas, rather than existence results, are often desirable. Rota, an expert in combinatorics, has developed a powerful approach to such problems: his methods manage to be both conceptual and computational at once. How does one achieve such a balancing act? By making the notation reflect the concepts. As a result Rota has become the Edgar Allen Poe of mathematics, exhuming Grassmann's regressive product from its premature burial.

Barnabei et al. motivate their ideas in terms of three important types of transformation: orthogonal transformations are essentially rotations in a multidimensional space; symplectic transformations arise from mechanics; and a general linear transformation is, as its name implies, the most general transformation that keeps straight lines straight. Barnabei et al. summarize the standard results in the first two cases as follows: (1) a Hilbert space is a vector space having a symmetric form $(x, y)$. That is, $(x, y)=(y, x)$. A transformation $T$ is orthogonal if it preserves this form, that is, $(T x, T y)=(x, y)$. Invariants under orthogonal transformations are polynomials in the symmetric form. Computations in Hilbert space are carried out in a naturally associated algebra, the Clifford algebra; (2) a symplectic space is a vector space having an antisymmetric (or symplectic) form $[x, y]$, that is, one such that $[y, x]=-[x, y]$. A transformation is symplectic if it preserves this form. Invariants under symplectic transformations are polynomials in the symplectic form. Computations occur in a naturally associated algebra, the Weyl algebra.

The parallel between these two theories is striking, and it suggests a fruitful question: is there an analogous setting for the general linear group? Barnabei et al. show that the answer is yes. They define a Peano space to be a vector space having a bracket $[x, y, z \ldots]$. Invariants under the general linear group are polynomials in the bracket. Computations are carried out in a naturally associated algebra, the double algebra. It is so called because it has two operations (rather than the usual one). They are, of course, modern versions of Grassmann's regressive and progressive product. Plus ça change...

Ideas that are ahead of their time do not always find immediate favour, and those that are not appreciated tend to be forgotten. But it is hard to keep a really good idea down. It was Grassmann's tragedy to be ahead of his time, but it is a mark of his genius that his ideas are now coming to full flower.

Ian Stewart is in the Mathematics Institute, University of Warwick, Coventry CV4 7AL, UK. 